\title{
Positivity-Preserving Runge-Kutta Discontinuous Galerkin Method on Adaptive Cartesian Grid for Strong Moving Shock
}

\author{
Jianming Liu' ${ }^{1,2}$, Jianxian Qiu ${ }^{3, *}$, Mikhail Goman ${ }^{2}$, Xinkai Li ${ }^{2}$ \\ and Meilin $\mathrm{Liu}^{4}$ \\ ${ }^{1}$ School of Mathematics and Statistics, Jiangsu Normal University, \\ Xuzhou 221116, China. \\ ${ }^{2}$ Faculty of Technology, De Montfort University, Leicester LE1 9BH, England. \\ ${ }^{3}$ School of Mathematical Sciences and Fujian Provincial Key Laboratory of \\ Mathematical Modeling and High-Performance Scientific Computation, \\ Xiamen University, Xiamen 361005, China. \\ ${ }^{4}$ Shanghai Institute of Satellite Engineering, Shanghai 200240, China
}

Received 01 June 2014; Accepted (in revised version) 16 January 2015

\begin{abstract}
In order to suppress the failure of preserving positivity of density or pressure, a positivity-preserving limiter technique coupled with $h$-adaptive Runge-Kutta discontinuous Galerkin (RKDG) method is developed in this paper. Such a method is implemented to simulate flows with the large Mach number, strong shock/obstacle interactions and shock diffractions. The Cartesian grid with ghost cell immersed boundary method for arbitrarily complex geometries is also presented. This approach directly uses the cell solution polynomial of DG finite element space as the interpolation formula. The method is validated by the well documented test examples involving unsteady compressible flows through complex bodies over a large Mach numbers. The numerical results demonstrate the robustness and the versatility of the proposed approach.
\end{abstract}

AMS subject classifications: 65M50; 65M60; 76L05

Key words: Discontinuous Galerkin method, adaptive Cartesian grid, positivity-preserving, immersed boundary method, complex geometry.

\section{Introduction}

Recently, the Cartesian grid methods have become very popular in computational fluid dynamics (see [1-11] and their references), because such methods do not suffer from the complex grid generation and grid management requirements which are inherent in other methods, and also these methods are easily extended to high order

${ }^{*}$ Corresponding author. Email address: jxqiu@xmu.edu.cn (J.-X. Qiu) 
numerical schemes. Conceptually, the Cartesian grid approach is much simpler to be implemented than other grid methods. In general, solid bodies with a Cartesian grid for the partition of the flow field are cut out of a single static background mesh and their boundaries are represented by different types of cut cells. When cut cells become very small, however, degenerate cells will be encountered. In this situation, numerical instability may occur when an explicit time step scheme is used in numerical calculations. Some techniques have already been employed to overcome these problems along with time step stability restrictions [1,6-9]. Although there are many different techniques, ghost cell method or immersed boundary method for its simplification still obtains many researcher's favourite $[2-5,10,11]$. In our recent article [12], we developed an adaptive Cartesian grid RKDG method combined with the ghost cell immersed boundary technique to deal with a complex geometry. This methodology was based on the image point ghost cell method [4] and used an inverse distance weighting interpolation formula to obtain the value at the image point. In this paper, we extend this idea and develop a new approach for immerse boundary treatment, in which the interpolation formula for cell solution polynomials is created on discontinuous Galerkin finite element space.

In practice, it is quite often to encounter the situation in which the density or pressure of the numerical solutions becomes negative [13-15]. For instance, highly energetic flows may contain regions with a dominant kinetic energy, and a relatively small internal energy which is easy to become negative in the simulation [16]. Another well-known example is the computational simulation of shock wave or gas detonation propagation through different geometries [15]. The shock diffraction may result in very low density and pressure [13-16]. In general, the most commonly used high order numerical schemes for solving Euler equations do not satisfy the positivity property, which may produce negative density or pressure and cause blow-ups of the numerical algorithm. The ad hoc methods in numerical strategy, which modify the computed negative density and/or the computed negative pressure to be positive, destroy not only a local and global conservation, but also often cause numerical instability [17]. Recently, based on certain Gauss-Lobatto quadratures and positivity-preserving flux, Zhang and Shu [13-15] used Lax-Friedrichs flux and successfully developed a positivity-preserving approach for high-order discontinuous Galerkin methods. Such an approach is also applied to unstructured meshes and $p$-adaptive numerical solutions by Kontzialis and Ekaterinaris [18]. In a recent paper of Wang et al. [16], they simplified the method and extended it to solve gaseous detonations. The aim of the present work, then, is to develop a simple approach under the adaptive Cartesian grid to simulate large Mach number flows with strong shock/obstacle interactions and shock diffraction. The present paper can be considered as a companion work to [12] on the so-called adaptive Cartesian grid RKDG methods for arbitrarily complex geometries. More specifically, in this paper we employed a simplified version of high-order positivity-preserving technique with $h$-adaptive RKDG method, and a modified version of the well-known Harten-Lax-van Leer contact numerical flux named as HLLC-HLL flux in [19] to remedy the numerical shock instability. 
The paper is organized as follows. In Section 2, the governing equations and their numerical discretization are presented, including the review of RKDG method, $h$ adaptive RKDG method and the limiting process near the discontinuous. The positivitypreserving limiter on adaptive Cartesian grid and the implementation of the ghost cell immersed boundary method coupling with the high-order accurate RKDG method are presented in Section 2. In Section 3, computational results are presented based on two dimension benchmark problems with complex geometries. The numerical results demonstrate the efficiency, robustness, and versatility of the proposed approach. Finally, some concluding remarks are given in Section 4.

\section{Governing equations and numerical method}

The inviscid compressible Euler equations can be written in vector form explicitly expressing the conservation laws of mass, momentum and energy, which are

$$
\frac{\partial U}{\partial t}+\nabla \cdot F=0
$$

where, the conservative state vector $U$ and the inviscid flux vectors $F=\left[f_{x}, f_{y}\right]^{T}$ are defined by

$$
U=\left[\begin{array}{c}
\rho \\
\rho u \\
\rho v \\
\rho E
\end{array}\right], \quad f_{x}=\left[\begin{array}{c}
\rho u \\
\rho u^{2}+p \\
\rho u v \\
u(\rho E+p)
\end{array}\right], \quad f_{y}=\left[\begin{array}{c}
\rho v \\
\rho u v \\
\rho v^{2}+p \\
v(\rho E+p)
\end{array}\right] \text {, }
$$

where $p, \rho, u$ and $v$ are variables, which represent the pressure, the density, and the two Cartesian components of the velocity vector, respectively, and $E$ is the total energy per unit mass. The pressure $p$ is obtained by using an equation of state for ideal gases

$$
p=(\gamma-1) \rho\left(E-\frac{1}{2}\left(u^{2}+v^{2}\right)\right)
$$

where $\gamma$ is the specific heat ratio.

\subsection{Review of RKDG method and $h$-adaptive RKDG method}

\subsubsection{RKDG method}

To formulate the RKDG method [20-24], we first discretize (2.1) in space using the discontinuous Galerkin method. For simplicity, $U$ is considered as a scalar function here only. If $U$ is vector-valued, one simply proceeds component by component. 
Assuming that $\mathbb{T}_{h}$ is a grid coverage of $\Omega$ where the domain $\Omega$ is subdivided into a collection of nonoverlapping elements $K$, we seek the approximate solution $U_{h}(t, x, y)$ in the finite element space of discontinuous functions defined as

$$
V_{h}=\left\{v_{h} \in L^{\infty}(\Omega):\left.v_{h}\right|_{K} \in P^{k}, \forall K \in \mathbb{T}_{h}\right\},
$$

where $P^{k}$ is a space of polynomials with degree $\leq k$.

Firstly, the equation (2.1) is multiplied by a test function $v(x, y) \in V_{h}$ and integrated over cell $K$, integration by parts is performed, and then the following semi-discrete form of (2.1) is obtained

$$
\frac{d}{d t} \int_{K} U(x, y, t) v d x d y-\int_{K} F(U) \cdot \nabla v d x d y+\sum_{i=1}^{N_{e}} \int_{e_{K}^{i}} F(U) \cdot \vec{n}_{i} v d s=0,
$$

where $\vec{n}_{i}$ is the outward unit normal to the edge $e_{K}^{i}$ and $N_{e}$ is the number of faces of the cell $K$. The volume integral term $\int_{K} F(U) \cdot \nabla v d x d y$ can be computed either exactly or by a numerical quadrature with sufficiently high order of accuracy. The line integral in (2.3) can also be discretized by a Gaussian quadrature with sufficiently high accuracy, that is

$$
\int_{e_{K}^{i}} F(U) \cdot \vec{n}_{i} v d s \approx\left|e_{K}^{i}\right| \sum_{\beta=1}^{q} \omega_{\beta} F\left(U\left(G_{i, \beta}, t\right)\right) \vec{n}_{i} v\left(G_{i, \beta}\right),
$$

where $q(=k+1)$ is the number of Gaussian quadrature points. Since the numerical solution $U_{h}$ is discontinuous on the interface between two elements, the flux $F\left(U\left(G_{i, \beta}, t\right)\right) \vec{n}_{i}$ must be replaced by a numerical flux function $\widehat{F}\left(U^{-}\left(G_{i, \beta}\right), U^{+}\left(G_{i, \beta}\right), \vec{n}_{i}\right)$, which depends on both the inner- and outer-trace of $U_{h}$ on $\partial K$, and the unit outward normal vector $\vec{n}_{e}$ of edge $e$. In this study, a modified robust HLLC numerical flux named as HLLC-HLL flux in [19] is used as the approximate Riemann solver. It should be emphasized that the choice of the numerical flux function is independent of the finite element spaces employed here.

To complete the definition of the RKDG method, the following third-order total variation diminishing (TVD) Runge-Kutta time discretization [25] is used to solve the semi-discrete form (2.3), that is

$$
\begin{aligned}
& u^{(1)}=u^{n}+\triangle t L\left(u^{n}, t^{n}\right), \\
& u^{(2)}=\frac{3}{4} u^{n}+\frac{1}{4} u^{(1)}+\frac{1}{4} \triangle t L\left(u^{(1)}, t^{n}+\triangle t\right), \\
& u^{n+1}=\frac{1}{3} u^{n}+\frac{2}{3} u^{(2)}+\frac{2}{3} \triangle t L\left(u^{(2)}, t^{n}+\frac{1}{2} \triangle t\right),
\end{aligned}
$$

where $L$ denotes the spatial discretization. For unsteady problems, global time steps are used in Runge-Kutta method, which are equal to the smallest local time step calculated by local maximum characteristic speed and the local cell size at each time level, which can be formulated by

$$
\Delta t_{\text {local }}=\mathrm{CFL} \frac{d x d y}{(|u|+a) d y+(|v|+a) d x} .
$$


Here, $a$ denotes the local sound speed; $d x$ and $d y$ denote the cell length.

\subsection{2. $h$-adaptive RKDG method}

In fact, for $h$-adaptive Cartesian grid RKDG method, apart from the appearance of hanging cell, there is no main difference from general RKDG method. The detailed procedure has been reported in our recent paper [12]. Cartesian grid in conjunction with tree data structure is a natural choice for solution adaptive grids. In this work, we use a generalized binary tree (e.g., quad-tree in 2D, octree in 3D, etc.) data structure. We do not allow any cells with side length ratio $>2$ or $<0.5$ to be neighbours. This restriction results in what is known as a balanced tree [26,27].

For the RKDG method, if a local basis of $P^{k}$ is given and denoted as $v_{j}^{(K)}(x, y)$ for $j=0, \cdots, Q_{k}$ in cell element $K$, then, numerical solution $U_{h}(x, y, t) \in V_{h}$ can be expressed as

$$
\left.U_{h}(x, y, t)\right|_{K}=\sum_{j=0}^{Q_{k}} U_{K}^{(j)}(t) v_{j}^{(K)}(x, y),
$$

where $U_{K}^{(j)}(t)\left(j=0, \cdots Q_{k}\right)$ are the degrees of freedom. Commonly, the local orthogonal basis functions are adopted during the implementation and numerical calculation of RKDG method, which are

$$
1, \phi_{1}, \phi_{2}, \phi_{1}^{2}-\frac{1}{3}, \phi_{1} \phi_{2}, \phi_{2}^{2}-\frac{1}{3}, \cdots,
$$

inside the rectangular element $K=\left[x_{K-\frac{1}{2}}, x_{K+\frac{1}{2}}\right] \times\left[y_{K-\frac{1}{2}}, y_{K+\frac{1}{2}}\right]$, and

$$
\phi_{1}=\frac{x-x_{K}}{\triangle x_{K} / 2}, \quad \phi_{2}=\frac{y-y_{K}}{\triangle y_{K} / 2},
$$

where $\left(x_{K}, y_{K}\right)$ is the centre of rectangle $K, \triangle x_{K}$ and $\triangle y_{K}$ are lengths of $K$ 's sides in the direction of $x$ and $y$, respectively. Obviously, $U_{K}^{(0)}(t)$ is the cell average of $U_{h}$ over $K$.

In order to retain the approximation accuracy and the local conservation character, a $L^{2}$ projection approach is used to obtain the new generated grid's degrees of freedom $[12,28]$. Suppose $U_{h}$ is already known on the mesh $\mathbb{T}_{h}\left(t_{n}\right)$, and we need to determine the degrees of freedom $U_{K^{\prime}}^{(j)}\left(t_{n}\right)\left(j=0, \cdots, Q_{k}\right)$ in a new cell $K^{\prime} \in \mathbb{T}_{h}\left(t_{n+1}\right)$. Let $U_{h}^{\prime}$ denote the $L^{2}$ projection of $U_{h}$, and satisfy the following equation

$$
\left.\int_{K^{\prime}} U_{h}^{\prime}\right|_{K^{\prime}} v_{j}^{\left(K^{\prime}\right)}(x, y) d x d y=\int_{K^{\prime}} U_{h} v_{j}^{\left(K^{\prime}\right)}(x, y) d x d y
$$

where $v_{j}^{\left(K^{\prime}\right)}(x, y)$ is a local basis of $P^{k}$ in cell element $K^{\prime}$. Substituting (2.7) into (2.8), we obtain the degrees of freedom in the new generated grid $K^{\prime}$. The implementation of the adaptive mesh for RKDG method based on $P^{2}$ polynomials on each element is 

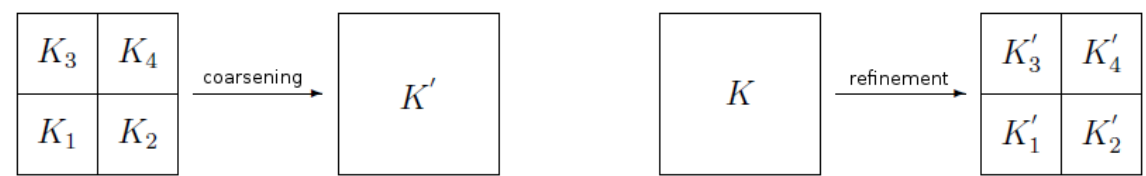

Figure 1: The sketches of coarsening (left) and refinement (right) in the adaptive mesh.

given in our recent paper [12]. Here, we only give the results directly. When four cells $K_{1}, K_{2}, K_{3}, K_{4}$ are flagged and coarsened to a new cell $K^{\prime}$ (see the left sketch in Fig. 1 ), the new degrees of freedom are given by

$$
\begin{aligned}
U_{K^{\prime}}^{(0)}= & \frac{1}{4}\left(U_{K_{1}}^{(0)}+U_{K_{2}}^{(0)}+U_{K_{3}}^{(0)}+U_{K_{4}}^{(0)}\right), \\
U_{K^{\prime}}^{(1)}= & \frac{1}{8}\left(U_{K_{1}}^{(1)}+U_{K_{2}}^{(1)}+U_{K_{3}}^{(1)}+U_{K_{4}}^{(1)}\right)+\frac{3}{8}\left(-U_{K_{1}}^{(0)}+U_{K_{2}}^{(0)}-U_{K_{3}}^{(0)}+U_{K_{4}}^{(0)}\right), \\
U_{K^{\prime}}^{(2)}= & \frac{1}{8}\left(U_{K_{1}}^{(2)}+U_{K_{2}}^{(2)}+U_{K_{3}}^{(2)}+U_{K_{4}}^{(2)}\right)+\frac{3}{8}\left(-U_{K_{1}}^{(0)}-U_{K_{2}}^{(0)}+U_{K_{3}}^{(0)}+U_{K_{4}}^{(0)}\right), \\
U_{K^{\prime}}^{(3)}= & \frac{1}{16}\left(U_{K_{1}}^{(3)}+U_{K_{2}}^{(3)}+U_{K_{3}}^{(3)}+U_{K_{4}}^{(3)}\right)+\frac{3}{16}\left(-U_{K_{1}}^{(2)}+U_{K_{2}}^{(2)}-U_{K_{3}}^{(2)}+U_{K_{4}}^{(2)}\right) \\
& \quad+\frac{3}{16}\left(-U_{K_{1}}^{(1)}-U_{K_{2}}^{(1)}+U_{K_{3}}^{(1)}+U_{K_{4}}^{(1)}\right)+\frac{9}{16}\left(U_{K_{1}}^{(0)}-U_{K_{2}}^{(0)}-U_{K_{3}}^{(0)}+U_{K_{4}}^{(0)}\right), \\
U_{K^{\prime}}^{(4)}= & \frac{1}{16}\left(U_{K_{1}}^{(4)}+U_{K_{2}}^{(4)}+U_{K_{3}}^{(4)}+U_{K_{4}}^{(4)}\right)+\frac{15}{32}\left(-U_{K_{1}}^{(1)}+U_{K_{2}}^{(1)}-U_{K_{3}}^{(1)}+U_{K_{4}}^{(1)}\right), \\
U_{K^{\prime}}^{(5)}= & \frac{1}{16}\left(U_{K_{1}}^{(5)}+U_{K_{2}}^{(5)}+U_{K_{3}}^{(5)}+U_{K_{4}}^{(5)}\right)+\frac{15}{32}\left(-U_{K_{1}}^{(2)}-U_{K_{2}}^{(2)}+U_{K_{3}}^{(2)}+U_{K_{4}}^{(2)}\right) .
\end{aligned}
$$

When a parent cell $K$ is flagged and refined to four child cells $K_{1}^{\prime}, K_{2}^{\prime}, K_{3}^{\prime}, K_{4}^{\prime}$, (see the right sketch in Fig. 1), the new degrees of freedom can be defined as

$$
\begin{aligned}
& U_{K_{i}^{\prime}}^{(0)}=U_{K}^{(0)}+a_{i} U_{K}^{(1)}+b_{i} U_{K}^{(2)}+a_{i} b_{i} U_{K}^{(3)}, \\
& U_{K_{i}^{\prime}}^{(1)}=\frac{1}{2} U_{K}^{(1)}+(-1)^{i} a_{i} b_{i} U_{K}^{(3)}+a_{i} U_{K}^{(4)}, \\
& U_{K_{i}^{\prime}}^{(2)}=\frac{1}{2} U_{K}^{(1)}+(-1)^{i}\left|a_{i} b_{i}\right| U_{K}^{(3)}+b_{i} U_{K}^{(5)}, \\
& U_{K_{i}^{\prime}}^{(3)}=\frac{1}{4} U_{K}^{(3)}, \quad U_{K_{i}^{\prime}}^{(4)}=\frac{1}{4} U_{K}^{(4)}, \quad U_{K_{i}^{\prime}}^{(5)}=\frac{1}{4} U_{K}^{(5)},
\end{aligned}
$$

where

$$
a_{i}=(-1)^{i} \frac{1}{2}, \quad b_{1}=b_{2}=-\frac{1}{2}, \quad b_{3}=b_{4}=\frac{1}{2} \quad(i=1,2,3,4) .
$$

It is noted that in the process of the grid refinement the cell averages in (2.10) for some physical variables, such as density and pressure, might appear to be negative. In this case, the cell averages of the new generated child cells are set to equal to the ones of their parents which doesn't destroy local mass conservation, and other moments of $U_{K^{\prime}}^{(j)}(t)\left(j=1, \cdots Q_{k}\right)$ are still calculated by the $L^{2}$ projection of $(2.8)$. 


\subsection{HLLC-HLL numerical flux}

In general, RKDG methods are in favour of Lax-Friedrichs flux [13, 14, 16, 20-24]. However, RKDG/Lax-Friedrichs flux is too dissipative for flows, such as turbulence flows with strong shocks computed in direct numerical simulations (DNS) and large eddy simulations (LES) [17]. In general, Harten-Lax-van Leer contact (HLLC) flux has smaller dissipation than Lax-Friedrichs flux $[29,30]$. However, it should be noted that original HLLC flux may produce shock instabilities in the vicinity of strong shocks [19]. Recently, using finite volume method with weighted average flux, Kim et al. [19] presented a robust HLLC flux named as HLLC-HLL flux to resolve these instabilities, which combined the HLLC and Harten-Lax-van Leer (HLL) schemes in a single framework with a switching function. In this study, we follow the idea of the switching function proposed in [19] and use the modified HLLC approximate Riemann solver (HLLC-HLL) as the numerical flux to remedy the numerical shock instabilities.

The HLL Riemann solver $[29,31]$ assumes a single constant state between two nonlinear waves, according to the reconstructed quantities - termed the left $(l)$ and right $(r)$ on the cell face, and the single constant state vector can be defined as

$$
U^{H L L}=\frac{S_{r} U_{r}-S_{l} U_{l}+F_{l}-F_{r}}{S_{r}-S_{l}}
$$

where $S_{l}$ is the smallest wave speed and $S_{r}$ is the largest wave speed [32]. The HLL Riemann solver can be written as

$$
\widehat{F}^{H L L}=\frac{S_{r} F_{l}-S_{l} F_{r}+S_{r} S_{l}\left(U_{r}-U_{l}\right)}{S_{r}-S_{l}} .
$$

Clearly, the original HLLC flux $[29,30]$ is a modification of the HLL scheme wherein the missing contacts and shear waves are resolved, which is given by

$$
\widehat{F}^{H L L C}= \begin{cases}F_{l}, & 0<S_{l} \\ F_{* l}=F_{l}+S_{l}\left(U_{* l}-U_{l}\right), & S_{l} \leq 0 \leq S_{*} \\ F_{* r}=F_{r}+S_{r}\left(U_{* r}-U_{r}\right), & S_{*} \leq 0 \leq S_{r} \\ F_{r}, & 0 \geq S_{r},\end{cases}
$$

where $U_{* l}$ and $U_{* r}$ are the conserved variable vectors in the star region separated by the contact, which are defined as

$$
U_{* k}=\rho_{k}\left(\frac{S_{k}-u_{n k}}{S_{k}-S_{*}}\right)\left[\begin{array}{c}
1 \\
S_{*} \\
u_{t k} \\
E_{k}+\left(S_{*}-u_{n k}\right)\left[S_{*}+\frac{p_{k}}{\rho_{k}\left(S_{k}-u_{n k}\right.}\right]
\end{array}\right], \quad k=l \text { or } r
$$


where the subscripts $n$ and $t$ represent the normal and tangential velocity components, and $S_{*}$ is the middle wave speed. $F_{* l}$ and $F_{* r}$ are obtained by applying RankineHugoniot conditions across each wave $[29,30]$. Because the HLL flux assumes a twowave system, $F_{* l}$ and $F_{* r}$ are the same as HLL flux given in (2.12).

In order to cure the shock instability in the vicinity of strong shocks, Kim et al. [19] introduced a switching function $f$, and the data of states $U_{* l}$ and $U_{* r}$ are replaced by $U_{* l}^{\text {new }}$ and $U_{* r}^{\text {new }}$ as follows

$$
\begin{aligned}
& U_{* l}^{\text {new }}=f \cdot U_{* l}+(1-f) \cdot U^{H L L}, \\
& U_{* r}^{\text {new }}=f \cdot U_{* r}+(1-f) \cdot U^{H L L} .
\end{aligned}
$$

The function $f$ has a value of 0 or 1 . Then the left and right fluxes $F_{* l}$ and $F_{* r}$ of (2.13) in the star region are defined by newly obtained data of states $U_{* l}^{\text {new }}$ and $U_{* r}^{\text {new }}$ as

$$
\begin{aligned}
& F_{* l}^{\text {new }}=F_{l}+S_{l}\left(U_{* l}^{\text {new }}-U_{l}\right), \\
& F_{* r}^{\text {new }}=F_{r}+S_{r}\left(U_{* r}^{\text {new }}-U_{r}\right) .
\end{aligned}
$$

In order to define the switching function $f$, we use a simple shock indicator $f_{p}$ :

$$
f_{p}= \begin{cases}1, & \text { if }\left|p_{r}-p_{l}\right|>\alpha\left|u_{n r}-u_{n l}\right| \sqrt{\gamma \rho_{l} p_{l}} \text { or }\left|p_{r}-p_{l}\right|>\alpha\left|u_{n r}-u_{n l}\right| \sqrt{\gamma \rho_{r} p_{r}} \\ 0, & \text { otherwise }\end{cases}
$$

presented in [33] to identify a shock wave. $\alpha$ is a constant, and is chosen as $\alpha=0.3$ in all calculations. The switching function $f$ is only employed when there is a strong shock, that is [19]

$$
\text { if } f_{p}=1, f= \begin{cases}1.0, & \text { if } M_{l}>1.0 \text { and } M_{r}>1.0 \\ 1.0, & \text { if } M_{l}<1.0 \text { and } M_{r}<1.0 \\ 0.0, & \text { otherwise }\end{cases}
$$

where $M_{l}$ and $M_{r}$ are local Mach numbers at the left and right cell face, respectively. In this way, when the function $f$ has a value 0 , the HLLC-HLL scheme will be switched to the HLL scheme. Furthermore, the HLLC-HLL flux is a positivity preserving flux. In fact, both HLL and HLLC fluxes are positivity-preserving [13,31,32]. As a consequence, the HLLC-HLL flux is a positivity preserving flux.

\subsection{Discontinuous detector}

If there are strong discontinuities in an approximate solution, oscillations may occur or even the method may break down. To enhance the stability of the method and eliminate possible spurious oscillations in the approximate solution, some kinds of limiting must be added during the time evolution. Unfortunately, most of limiters frequently 
identify regions near smooth extrema as requiring limiting and this typically results in a reduction of the optimal high-order convergence rate. In this study, discontinuity detector introduced by Krivodonova et al. in [34] is used to distinguish regions where solutions are smooth or discontinuous. With such knowledge, limiting would only be used near discontinuities, hence, high-order accuracy for the solution would be preserved in smooth regions.

The discontinuity detector works in the following way. First partition the cell boundary $\partial K$ into two portions $\partial K^{-}$and $\partial K^{+}$, where the flow is into $(V \cdot \vec{n}<0, \vec{n}$ is the normal vector to $\partial K)$ and out of $(V \cdot \vec{n}>0)$ cell $K$, respectively. Then the discontinuity detector is defined as

$$
I_{K}=\frac{\left|\int_{\partial K^{-}}\left(U_{K}-U_{n b K}\right) d s\right|}{h^{(k+1) / 2}\left|\partial K^{-}\right| \mid U_{K} \|}
$$

and if $I_{K}>1, U_{K}$ is discontinuous; otherwise, $U_{K}$ is smooth. Note that here $h$ is the radius of the circumscribed circle in element $K$, and $\left\|U_{K}\right\|$ is a maximum norm based on local solution maximum at integration points. Considering its abilities both for the shock and the contact discontinuity, we use entropy as the discontinuity detection variable for Euler equations [34]. Furthermore, a local slope limiting named as TVB limier introduced in $[22,23]$ is used near the discontinuity region. In the original TVB limiter, TVB limiter constant $M$ is taken to prohibit the degeneracy of accuracy at the smooth extrema and make the resulting RKDG scheme retains its optimal accuracy. The TVB limiter will not destroy accuracy for large enough $M$, see [22,23] for more details of the TVB limiter. In this study, we first use a discontinuity detector introduced to distinguish regions where solutions are smooth or discontinuous, then we use the TVB limiter at these cells which have been detected as discontinuous cells. In the present study, except the special instructions, $M=50$ is used in all examples. For uniform grids, there are no trouble in the limiting procedure, however, for $h$-adaptive Cartesian grid, the appearances of hanging nodes would make some neighbours not real cells with same refinement levels, for the detailed descriptions, which can refer to our recent paper [12].

\subsection{Positivity-preserving method}

The solutions of the density and the pressure computed by any numerical methods should be positive. However, for strong shocks and through different geometries, numerical solutions may produce negative pressures or densities. In this section, we will develop a positivity-preserving method and implement it with $h$-adaptive Cartesian grid RKDG methods.

Positivity limiters are constructed based on the observation of Jensen inequality. Because the pressure $p$ is a concave function of the state variables. For

$$
U_{1}=\left[\rho_{1}, \rho_{1} u_{1}, \rho_{1} v_{1}, \rho_{1} E_{1}\right]^{T}, \quad U_{2}=\left[\rho_{2}, \rho_{2} u_{2}, \rho_{2} v_{2}, \rho_{2} E_{2}\right]^{T},
$$


Jensen inequality [16] implies

$$
p\left(s U_{1}+(1-s) U_{2}\right) \geq s p\left(U_{1}\right)+(1-s) p\left(U_{2}\right) .
$$

for every $0 \leq s \leq 1$.

Based on certain Gauss-Lobatto quadratures and positivity preserving flux, in order to preserve the positivity of density and pressure fields in numerical calculations, a limiting procedure under a CFL-like condition $[17,36]$ presented by Zhang and Shu [13-15] and simplified by Wang et al. [16] is developed in limiting the solution with positivity-preserving flux and the TVB limiter.

Given the DG polynomials

$$
U_{K}(x, y)=\left[\rho_{K}(x, y),(\rho u)_{K}(x, y),(\rho v)_{K}(x, y),(\rho E)_{K}(x, y)\right]^{T}
$$

with its cell average $\bar{U}_{K}^{n}=\left[\bar{\rho}_{K}^{n}, \overline{\rho u}_{K}^{n}, \overline{\rho v}_{K}^{n}, \overline{\rho E}_{K}^{n}\right]^{T}$ and the assumption of the positive cell averages of the density and pressure at $n$-th time step, the first step is to limit the coefficients for the density field and then the second step is to limit the pressure and enforce the positivity of the pressure. Here the assumption for the cell averages of the density and pressure are positive at $n$-th time step. This procedure is accomplished by setting a fixed small value $\epsilon$, for example, $\epsilon=10^{-13}$. Here, based on the positivity-preserving limiter under unstructured grid in [15], we use the improved pressure positivity-preserving limiter [16] to give a simple algorithm as follows:

- In each cell, modify the density first:

- if $\bar{\rho}_{K}^{n} \geq \epsilon$, then replace $\rho_{K}(x, y)$ by

$$
\widehat{\rho}_{K}(x, y)=\theta_{1}\left(\rho_{K}(x, y)-\bar{\rho}_{K}^{n}\right)+\bar{\rho}_{K}^{n}, \theta_{1}=\min \left(\left|\frac{\bar{\rho}_{K}^{n}-\epsilon}{\bar{\rho}_{K}^{n}-\rho_{\min }}\right|, 1\right),
$$

and set

$$
\widehat{U}_{K}(x, y)=\left[\widehat{\rho}_{K}(x, y),(\rho u)_{K}(x, y),(\rho v)_{K}(x, y),(\rho E)_{K}(x, y)\right]^{T},
$$

where $\rho_{\min }$ is the minimum value of densities looping over the set of the Gauss-Lobatto quadrature points $S_{K}$. In practice, for the local orthogonal bases (2.7), the final degrees of freedom $U_{K}^{(j)},\left(j=1, \cdots, Q_{k}\right)$ for the density expansion are only modified by $\theta_{1} U_{K}^{(j)}$.

- if $\bar{\rho}_{K}^{n}<\epsilon$, set

$$
\widehat{U}_{K}(x, y)=\left[\bar{\rho}_{K}^{n},(\rho u)_{K}(x, y),(\rho v)_{K}(x, y),(\rho E)_{K}(x, y)\right]^{T} .
$$


- Then modify the pressure:

- if $\bar{p}_{K}^{n} \geq \epsilon$, set

$$
\theta_{2}=\min _{X \in S_{K}} \theta_{X}, \theta_{X}= \begin{cases}\frac{p\left(\bar{U}_{K}^{n}\right)-\epsilon}{p\left(\bar{U}_{K}^{n}\right)-p\left(\widehat{U}_{K}(X)\right)}, & \text { if } p\left(\widehat{U}_{K}(X)\right)<\epsilon \\ 1, & \text { if } p\left(\widehat{U}_{K}(X)\right) \geq \epsilon\end{cases}
$$

and have the following new vector of polynomials

$$
\widehat{\widehat{U}}_{K}(x, y)=\theta_{2}\left(\widehat{U}_{K}(x, y)-\bar{U}_{K}^{n}\right)+\bar{U}_{K}^{n} .
$$

- if $\bar{p}_{K}^{n}<\epsilon$, set $\widehat{\widehat{U}}_{K}(x, y)=\bar{U}_{K}^{n}$.

- Replace $U_{K}(x, y)$ by $\widehat{\widehat{U}}_{K}(x, y)$ for each cell $K$ in the DG solutions.

In the original positivity-preserving limiter in $[13,15]$, a quadratic equation of $\theta_{X}$ should be solved, in practice the solved $\theta_{X}$ cannot guarantee the strict non-negativity of pressure numerically due to the round off errors for some wild data [16]. The limiter developed here for pressure is different from the one in paper [16]. Here, in order to suppress zero pressure, $s p\left(U_{1}\right)+(1-s) p\left(U_{2}\right)$ in (2.20) is set equal to $\epsilon$ to calculate the value $\theta_{X}$. Furthermore, if $\bar{p}_{K}^{n}<\epsilon$, we directly ask $\widehat{\widehat{U}}_{K}(x, y)=\bar{U}_{K}^{n}$ which is suggested in [15] for unstructured grid.

\subsection{Solution-based mesh refinement on Cartesian grids}

Sensors are employed in this paper to detect and localize physical flow phenomena [35]. Because the divergence of velocity is direction independent and very effective in locating shock and the gradient of density finds shock and contact discontinuity well, we consider a sensor combined with divergence of velocity and gradient of density as follows

$$
\tau_{d i}=|\nabla \cdot V| d_{i}^{\frac{3}{2}}, \quad \tau_{g i}=|\nabla \rho| d_{i}^{\frac{3}{2}}
$$

for $i=1,2, \cdots, N_{c}$, where $N_{c}$ is the total number of cells and $d_{i}=\sqrt{|K|}(|K|$ is the cell volume). The standard deviation of both parameters are computed as

$$
\sigma_{d}=\sqrt{\frac{\sum_{i=1}^{N_{c}} \tau_{d i}^{2}}{N_{c}}}, \quad \sigma_{g}=\sqrt{\frac{\sum_{i=1}^{N_{c}} \tau_{g i}^{2}}{N_{c}}} .
$$

A cell is flagged for refinement or coarsening if one of two possible conditions hold: 
(a) if either $\tau_{d i}>\omega_{1} \sigma_{d}$ or $\tau_{g i}>\omega_{2} \sigma_{g}$, the cell is flagged for refinement

(b) if both $\tau_{d i}<\omega_{3} \sigma_{d}$ and $\tau_{g i}<\omega_{4} \sigma_{g}$ the cell is flagged for coarsening

where $\omega_{l}(l=1,2,3,4)$ are adjustable coefficients based on different problems. $\omega_{1}, \omega_{2}$ can be chosen between $1.0 \sim 1.5$ and $\omega_{3}, \omega_{4}$ can be chosen between $0.1 \sim 0.4$. In our tests, we set $\omega_{1}=\omega_{2}=1.2$ and $\omega_{3}=\omega_{4}=0.3$.

\subsection{Boundary treatment}

In order to implement the developed numerical method to simulate the flow over arbitrary complex solid bodies on Cartesian grid, the boundary cut-cell intersected by the solid surface must be treated specifically. In this study, the ghost cell immersed boundary method is used. During the process, we need to proceed by identifying cells whose centers are inside the solid, and then name them as the ghost cells. Solid cells can be identified by ray-tracing approach. Actually, we only need to identify the closest row of solid cells near the body surface as the ghost cells $(g c)$, because the stencil of RKDG is compact and demand only the nearest neighbour cells, which is shown in Fig. 2. It is easy to derive a numerical scheme that allows us to calculate the value of each variable at the ghost cell center such that the boundary conditions on the immersed boundary are satisfied. Here, we propose the following equations to be used to calculate the values on the ghost cells for two dimensional inviscid flow

$$
\begin{aligned}
& p_{g c}=p_{r p}-\rho_{r p} \frac{V_{\overrightarrow{t r p}}^{2}}{R} \Delta n, \\
& \rho_{g c}=\rho_{r p}, \quad V_{\vec{t} g c}=V_{\vec{t} r p}, \\
& V_{\vec{n} g c}=-\frac{\delta_{g}}{\delta_{r}} V_{\vec{n} r p},
\end{aligned}
$$

where $R$ is the local radius of curvature of the wall; $V_{\vec{t}}$ and $V_{\vec{n}}$ are the two velocity components on tangential and normal to the body surface, respectively; $r p$ denotes the reference point chosen along the normal direction to the body surface from the ghost cell ( $g c$; see Fig. 2); $\Delta n=\delta_{g}+\delta_{r}$ indicates the distance between the $r p$ and $g c$; and $\delta_{g}$ and $\delta_{r}$ denote the distances from the ghost point $g c$ and the reference point $r p$ to the wall, respectively. In the current study, $\delta_{r}$ is set to the length of ghost cell diagonal. Clearly, the nonpenetration boundary conditions are satisfied automatically. Instead of utilizing image point as shown in our recent paper [12], we use reference point to set the boundary, in which the reference point can be located in a fluid cell. Therefore, we can directly use the cell solution polynomial of DG finite element space as the interpolation formula to obtain the value at the reference point. That is an advantage of DG method. In the finite volume method or finite difference method, an interpolation must be set up to calculate the values at the reference point. For instance, based on the finite volume method, in the paper [37], the primitive variables at a reference point are interpolated from the primitive variables of the three closest neighbor cell centers using the linear interpolation. However, if an image point is used 


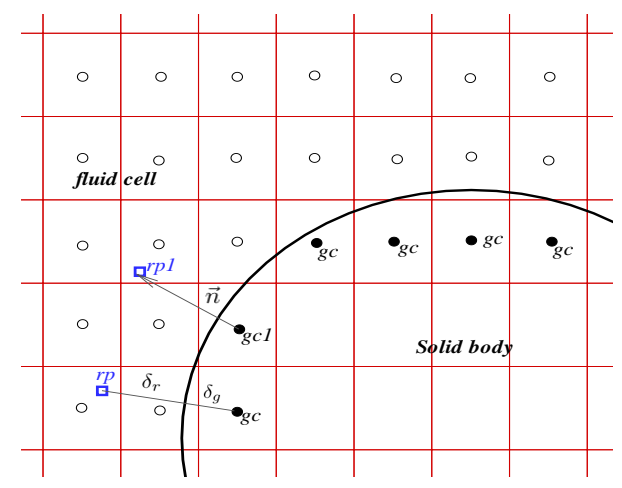

Figure 2: 2D schematic describing ghost cell method used in the current solver.

in DG method, a situation may be encountered where the image point may locate in the ghost cell itself. In this situation, there is no ready-made polynomial to interpolate the value at the image point.

In order to test the approximated accuracy, we compare the reference point method (2.21) with the image point method (with a change of $r p$ to the image point of $i p$ in the equation (2.21)) by a subsonic flow at Mach number 0.38 around a circular cylinder with radius $r=0.5$. As the setting in the paper [12], we consider the same background grid with three different initial mesh refinement numbers $\left(N_{r}=1,2,3\right)$. As a sample, the local view of the computational grid at $N_{r}=3$ and the Mach contours are shown in Fig. 3. From the Table 1, we can find the entropy errors by the present reference point method (2.21) are smaller than the ones by the image point method.

Table 1: $L^{\infty} / L^{2}$ errors in entropy for the circular cylinder.

\begin{tabular}{|ccccc|}
\hline & \multicolumn{2}{c}{ Image point method } & \multicolumn{2}{c|}{ Reference point method } \\
\hline$N_{r}$ & $L^{\infty}\left(\varepsilon_{\text {ent }}\right)$ & $L^{2}\left(\varepsilon_{\text {ent }}\right)$ & $L^{\infty}\left(\varepsilon_{\text {ent }}\right)$ & $L^{2}\left(\varepsilon_{\text {ent }}\right)$ \\
\hline 1 & $8.9412 \mathrm{e}-03$ & $4.4780 \mathrm{e}-03$ & $3.4121 \mathrm{e}-03$ & $1.4635 \mathrm{e}-03$ \\
2 & $4.5468 \mathrm{e}-03$ & $1.6233 \mathrm{e}-03$ & $1.2699 \mathrm{e}-03$ & $4.2361 \mathrm{e}-04$ \\
3 & $2.2591 \mathrm{e}-03$ & $5.4040 \mathrm{e}-04$ & $3.7106 \mathrm{e}-04$ & $1.2013 \mathrm{e}-04$ \\
\hline
\end{tabular}

\section{Numerical examples}

In this section, numerical experiments are carried out to demonstrate the effectiveness of adaptive Cartesian grid RKDG method with positivity-preserving method developed in this paper. For these typical numerical test cases, the original high-order RKDG methods may fail to preserve the positivity of density or pressure. Here, the 


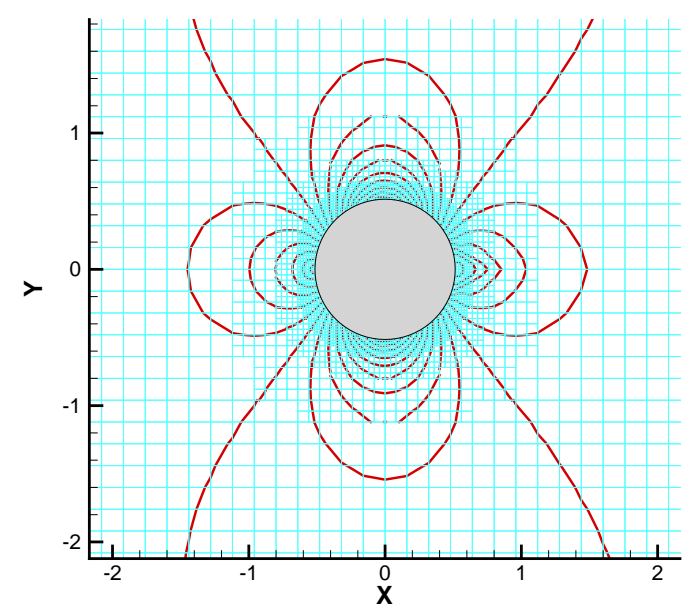

Figure 3: Local view of the computational grid at $N_{r}=3$ and the Mach contours.

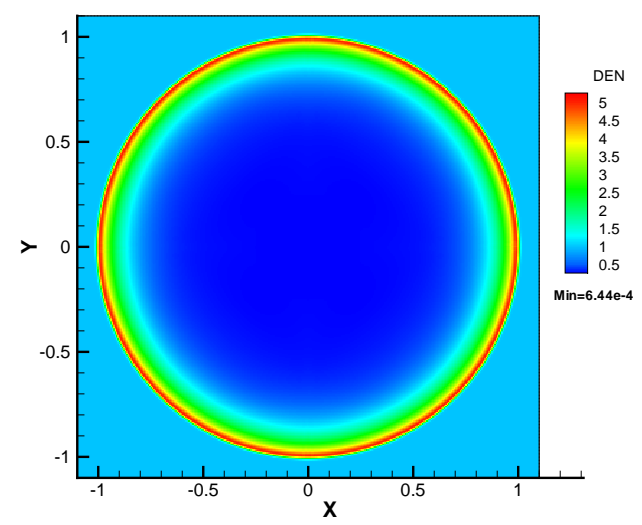

(a) Density

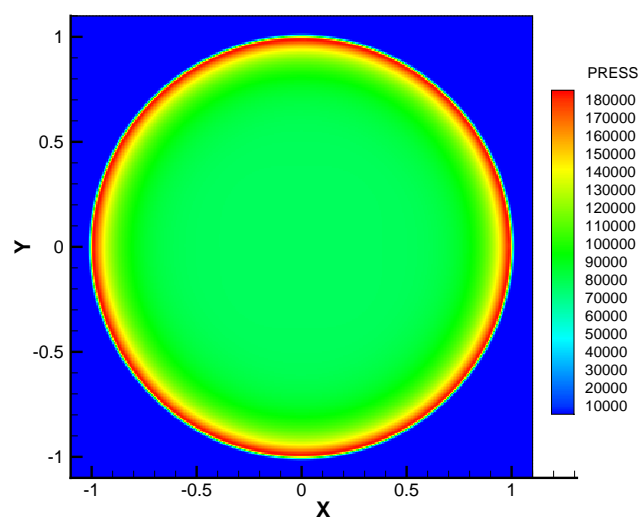

(b) Pressure

Figure 4: Density and pressure contour images with positivity-preserving limiter for Sedov blast wave.

$h$-adaptive RKDG method with $P^{2}$ polynomial is used in each cell for all numerical test examples. The testes here use a smaller CFL number 0.034 to satisfy the limited CFL number $[13,15]$.

\subsection{Two-dimensional Sedov blast wave}

The first example is a two-dimensional Sedov problem which was studied in [13, $15,16]$, in which the computation is performed on the domain $[-1.1,1.1] \times[-1.1,1.1]$. In this case, a high pressure region occupies in the computational cells near the origin. 


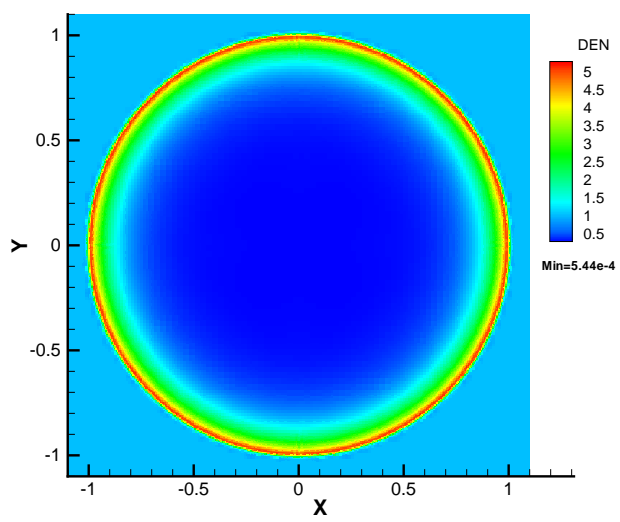

(a) Density

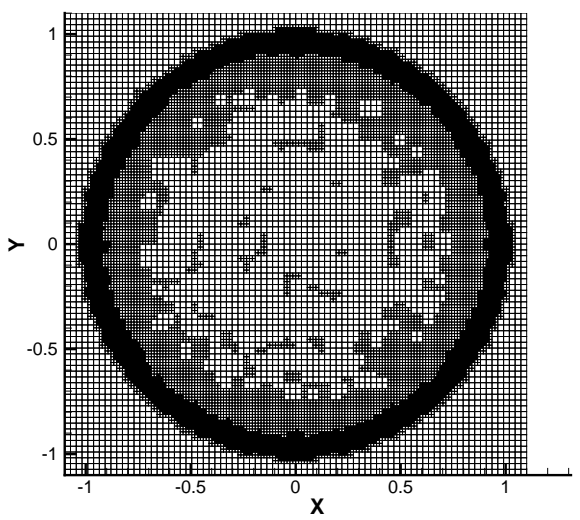

(c) Solution adaptive Cartesian grid

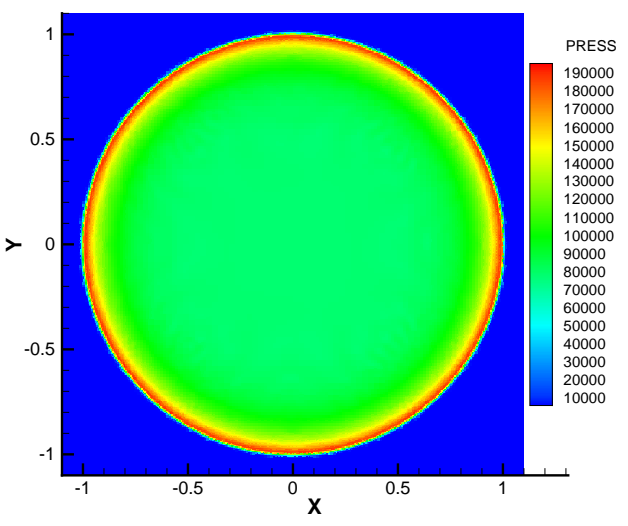

(b) Pressure

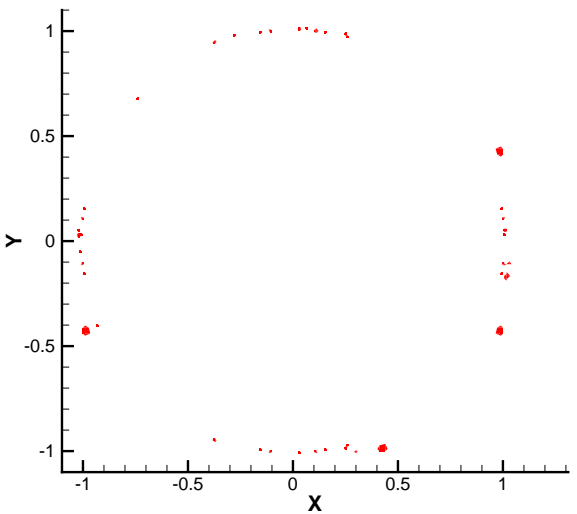

(d) The position acted by the posivity-preserving limiter for TVB limiter $M=50$

Figure 5: Density and pressure contour images with positivity-preserving limiter for Sedov blast wave, solution adaptive Cartesian grid: a base grid of $80 \times 80$ with two levels of mesh refinement, and the position operated by the posivity-preserving limiter for the TVB limiter $M=50$.

An initial condition is given by

$$
(\rho, u, v, p)= \begin{cases}\left(1,0,0,4 \times 10^{-13}\right) & \text { if }|x|>\Delta x,|y|>\Delta y \\ \left(1,0,0, \frac{9.79264}{\Delta x \Delta y} \times 10^{4}\right) & \text { otherwise }\end{cases}
$$

where $\Delta x=\Delta y=1.1 / 160$. A final time is $t=1.0 \times 10^{-3}$. An outflow condition is also applied on the computational boundary.

In this special case, we first use a uniform grid and set $\Delta x=\Delta y=1.1 / 160$ with TVB limiter parameter $M=8000$. Without the positivity limiter, the DG scheme is blown up for such a large value of $M$. However, if the positivity-preserving method is 


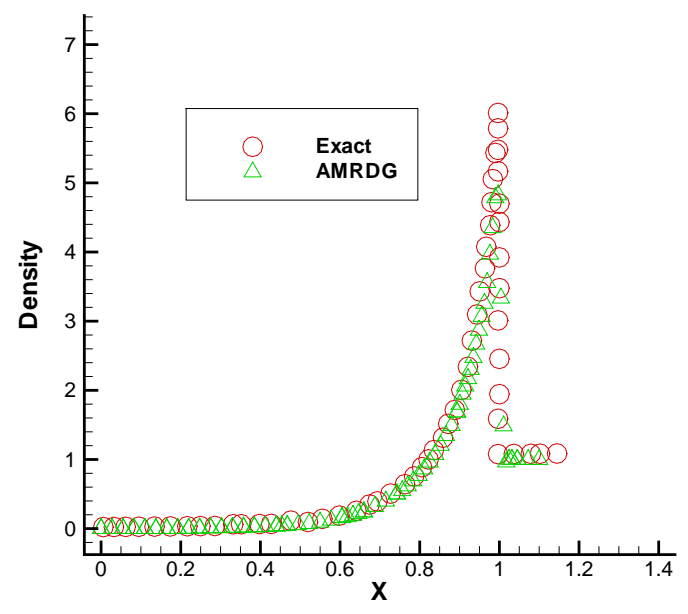

Figure 6: Density profile along $y=0$.

used to simulate this case, the computational results are satisfied and are compatible with ones presented in $[13,15,16]$. Fig. 4 shows the contour images of the density and pressure with positivity-preserving limiter for Sedov blast wave. Furthermore, the example is also tested with $h$-adaptive Cartesian grid RKDG method, in which a base grid of $80 \times 80$ is used with two levels of mesh refinements. Fig. 5 shows the contour images of density and pressure with positivity-preserving limiter for Sedov blast wave using the adaptive Cartesian grids with two levels of mesh refinement. The computational results demonstrate that the adaptive grid method presented in this paper does not make the solutions blown up even when the mesh grids are refined with a large value of $M$. That is clearly shown in Fig. 6, where the numerical density agrees well with the exact solution. In order to demonstrate whether the presented schemes are positivity preserving, the calculation at the TVB limiter $M=50$ is also tested. If the positivity preserving limiter is not used, for $M=50$, the solution evolution broke up and the negative pressure or density appeared. In this test, the position operated by the posivity-preserving limiter for the TVB limiter $M=50$ at time $t=1.0 \times 10^{-3}$ is shown in Fig. 5(d).

\subsection{High Mach number astrophysical jet}

The second example is a high Mach number astrophysical jet problem, which was used as an example in $[13,15,36]$.

In this example a Mach 80 jet problem with $\gamma=5 / 3$ is computed. The computation is performed on the domain $[0,2] \times[-0.5,0.5]$, which is full of the ambient gas with $(\rho, u, v, p)=(5,0,0,0.4127)$ initially. The boundary conditions for the right, the top and the bottom are outflow. For the left boundary, $(\rho, u, v, p)=(5,30,0,0.4127)$ if 


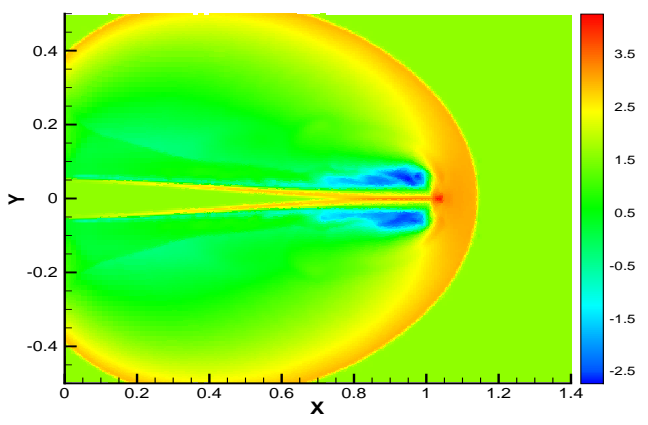

(a) Density

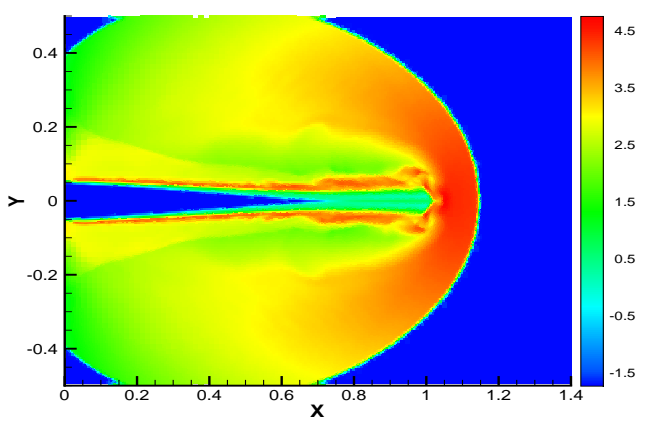

(c) Temperature

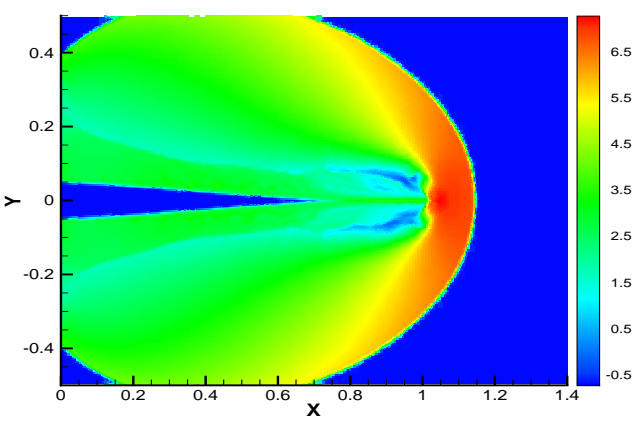

(b) Pressure

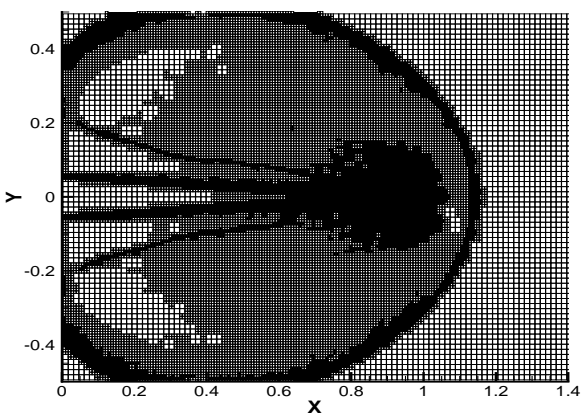

(d) Solution adaptive Cartesian grid

Figure 7: Simulation of Mach 80 jet with a simple positivity-preserving limiter. Scales are logarithmic.

$y \in[-0.05,0.05]$ and $(\rho, u, v, p)=(5,0,0,0.4127)$ otherwise. The terminal time is 0.07. The computation is performed with $\Delta x=\frac{2.2}{150}$ and $\Delta y=\frac{1.1}{80}$ combined with two levels solution refinements. TVB limiter parameter is $M=10000$. Without the positivitypreserving limiter, the DG scheme will blow up for such large value of $M$. Fig. 7 shows the contour images for the density, pressure and temperature. One can observe that these results are in very good agreement with these in $[13,15]$. Clearly, the solutions with the adaptive Cartesian grid method developed in this paper capture well with the flow character as shown in Fig. 7(d).

\subsection{A shock wave diffracts at a convex corner}

A shock wave diffracting at a sharp convex corner is a benchmark problem in computational fluid dynamics. When the Mach number of the shock wave becomes larger, low density or pressure may appear [15].

Zhang and Shu in [15], used positivity-preserving high order discontinuous Galerkin schemes on triangular meshes to simulate a Mach 10 shock diffracting at a $120^{\circ}$ convex corner. In this paper, we use the same example, in which an initial condition is a pure right-moving shock of Mach=10, initially located at $x=3.4$ and $6 \leq y \leq 11$, mov- 


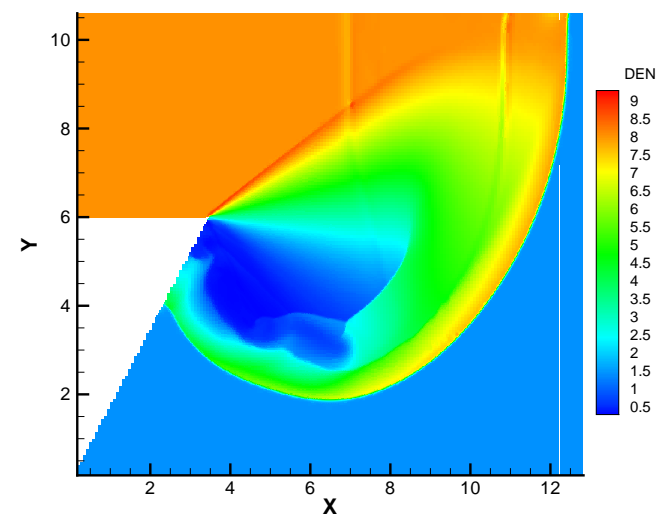

(a) Density

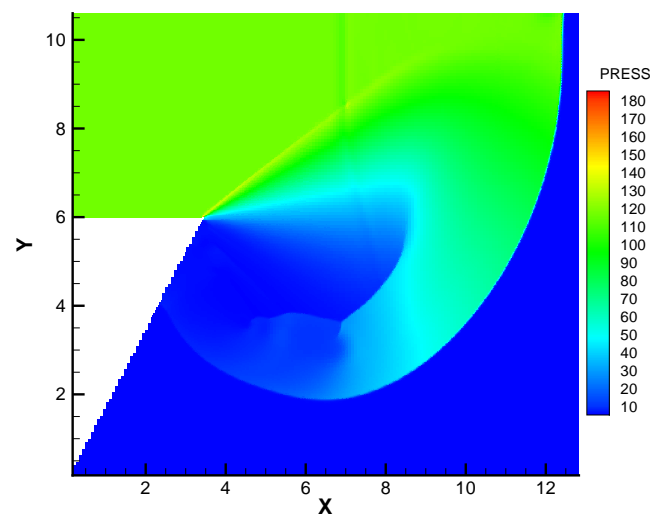

(b) Pressure

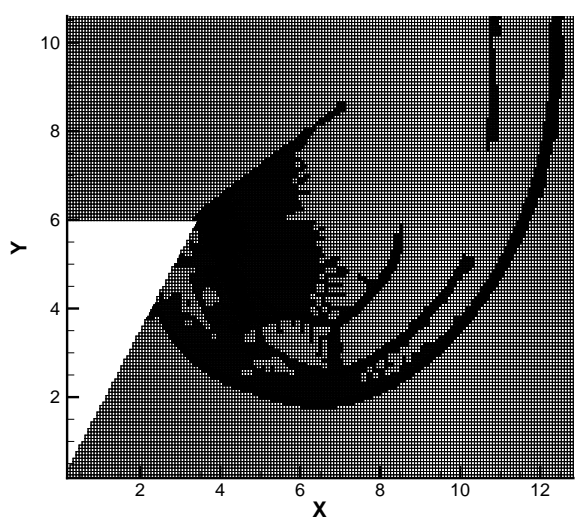

(c) Solution adaptive grid

Figure 8: A shock wave diffracts at a convex corner with a simple positivity-preserving limiter at time $t=0.9$.

ing into undisturbed air ahead of the shock with a density of $\rho=1.4$ and pressure of $p=1.0$. The terminal time is $t=0.9$. The computation is performed at $\Delta x=\frac{13.4}{180}$ and $\Delta y=\frac{11.4}{154}$ with two levels solution refinements.

In this geometry, a special procedure will be encountered, which is the problem of multi-valued ghost points. It is often found near unresolved thin surfaces and sharp corners, such as the cases at the sharp trailing edge of an airfoil or near the apex of triangle or near the convex corner as shown in Fig. 8. At sharp corners, one cell centre inside the geometry may be the ghost cell center for one side of the corner surface, as well as for the other sides. Also, a ghost cell center pertaining to one side of a corner surface may be located inside the flow field on the other side of the corner. To handle this case, we firstly find the fluid cells, in which the multiple valued ghost points are 


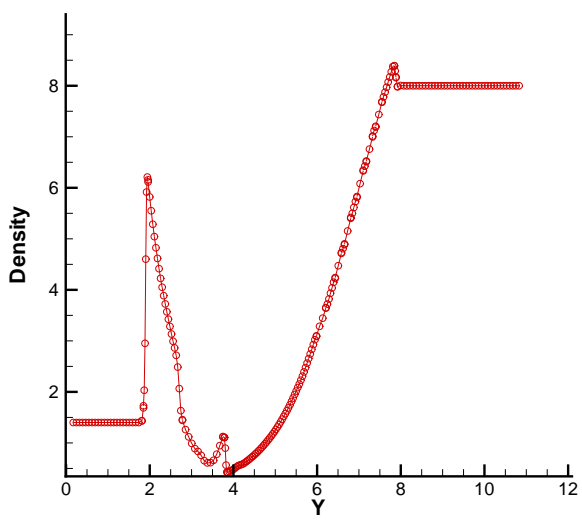

(a) Density $(x=6)$

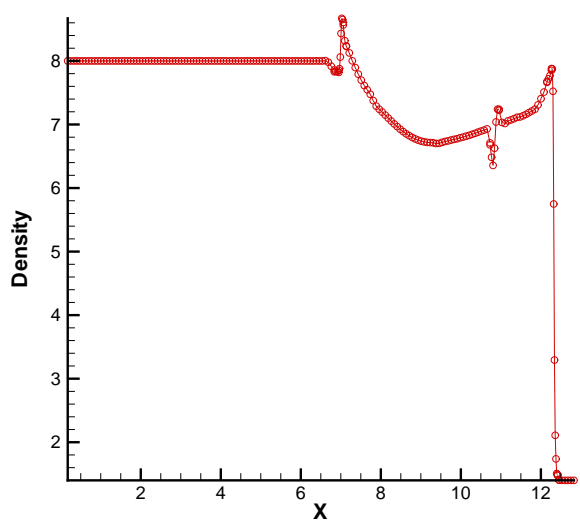

(c) Density $(y=8.5)$

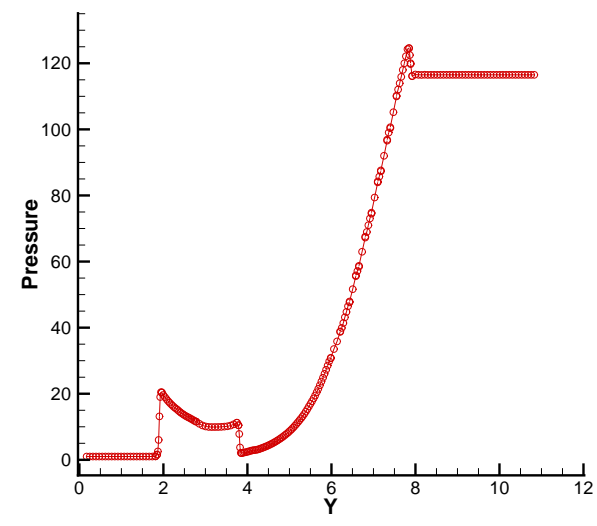

(b) Pressure $(x=6)$

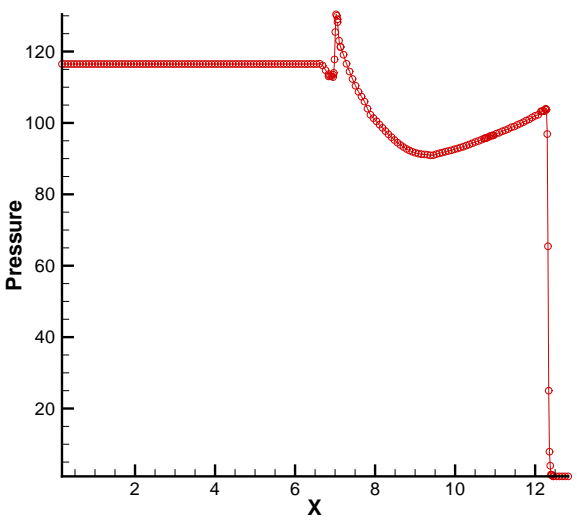

(d) Pressure $(y=8.5)$

Figure 9: Plots of density and pressure along the lines: $x=6$ and $y=8.5$ at time $t=0.9$.

needed to advance the solution process, and then set flags. Generally speaking, the ghost boundary conditions are given before time evolution. In this special situation, the multiple valued ghost points are evaluated during the time evolution. The rest fluid cells are computed as usual. The advantage of this approach is that there is no need to allocate new memory to save the values on the multiple valued ghost points.

For this problem, the density and pressure in the region across the convex corner will drop close to zero. The high order DG method without the positivity-preserving limiter may blow up the solutions [15]. However, when the adaptive grid techniques developed in this paper are implemented, the numerical solutions look very good and stable as shown in Fig. 8. When right-moving shock passes through the sharp convex corner, many complicated flow characteristics, such as contact discontinuity and rarefactional wave, will appear. These flow characteristics can be apparently found in 


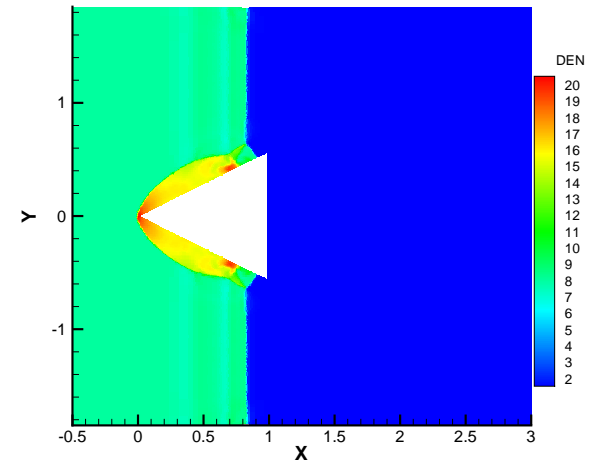

(a) Density at $t=0.08$

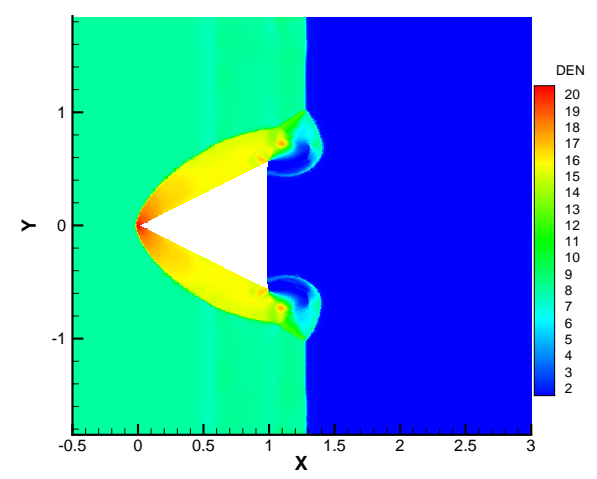

(c) Density at $t=0.125$

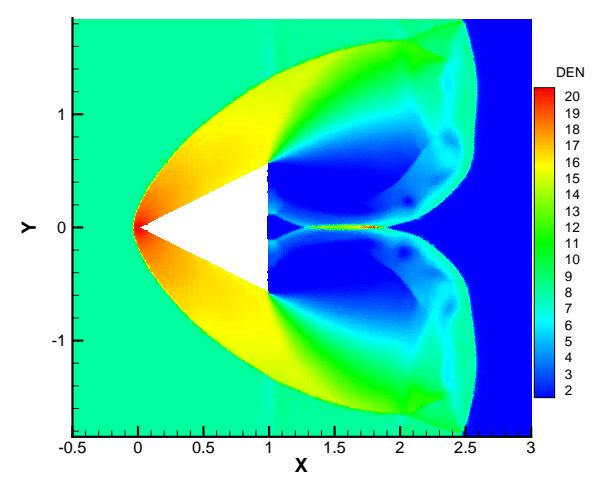

(e) Density at $t=0.245$

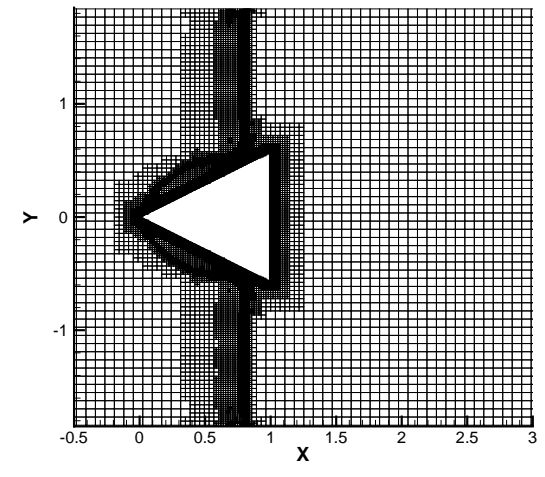

(b) Grid at $t=0.08$

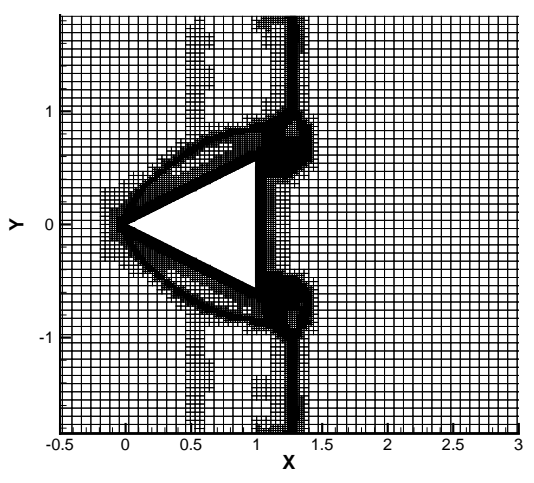

(d) Grid at $t=0.125$

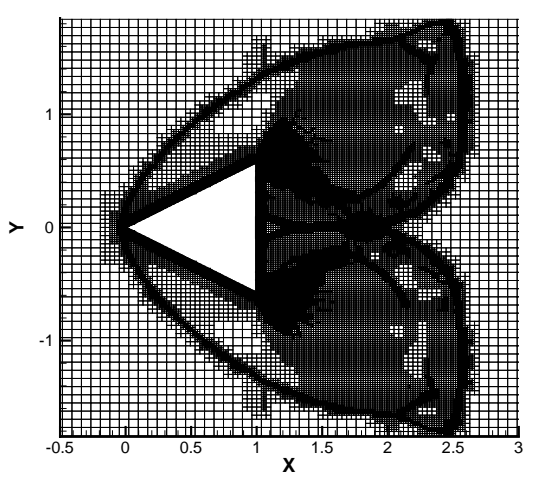

(f) Grid at $t=0.245$

Figure 10: A shock wave pass a finite wedge with a simple positivity-preserving limiter at different time.

Figs. 8(a) and 8(b). That is justified by the line plots of Figs. 9(a) and 9(b) for the density and pressure along a line at $x=6$, which demonstrate that solutions capture well 


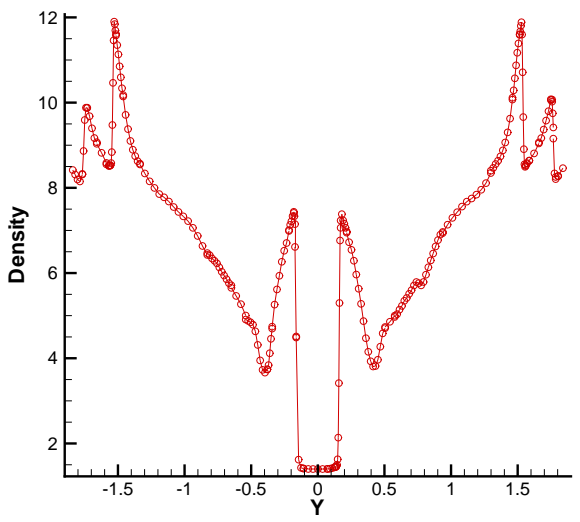

(a) Density $(x=2.25)$

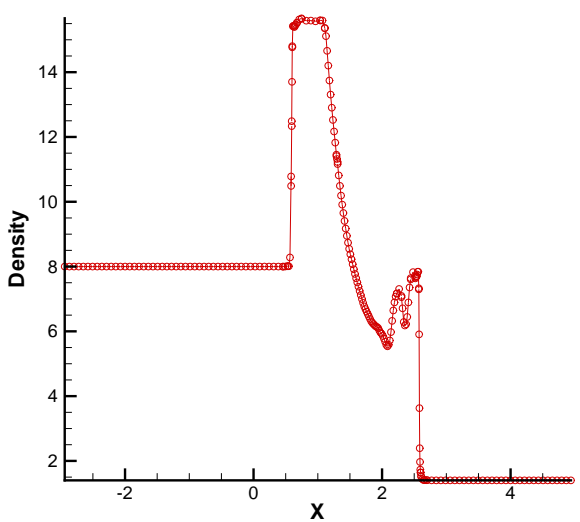

(c) Density $(y=1)$

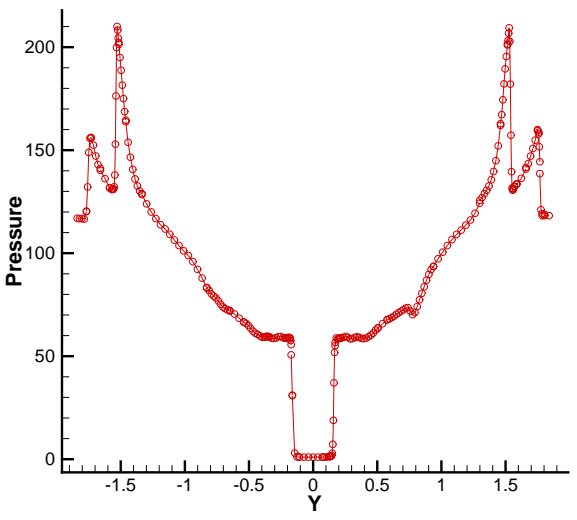

(b) Pressure $(x=2.25)$

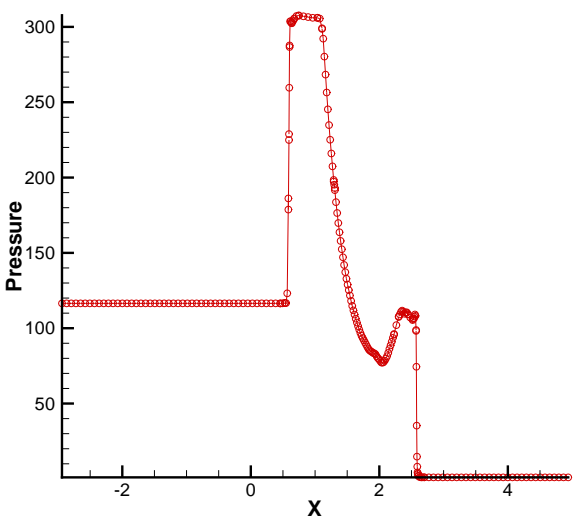

(d) Pressure $(y=1)$

Figure 11: Plots of density and pressure along the lines: $x=2.25$ and $y=1$ at time $t=0.245$.

for the contact discontinuities. Furthermore, Fig. 8(c) shows that the adaptive sensors defined in Section 3 is effective to capture the contact discontinuities and shocks. The line plots of the density and pressure along a line at $y=8.5$ are also plotted in Figures 9(c) and 9(d), where we can apparently find that there are a weak shock, a contact discontinuity and a strong shock, respectively. However, the weak shock and the contact discontinuity are too dissipate and have not being sharply captured by the numerical solution given in [15] with triangular elements (Mesh size is 0.05).

\subsection{Schardins problem: shock waves pass a finite wedge}

In order to show the ability of the present $h$-adaptive RKDG method for complex geometry, a Mach 10 shock passing a triangle is considered. In this example, the initial condition is a pure right-moving shock of Mach $=10$, initially located at $x=0.2$, moving 
into undisturbed air ahead of the shock with a density of $\rho=1.4$ and pressure of $p=1.0$. The set up for this test case is identical to the test case presented in [15]. It is interesting to note that in this example the density and the pressure in the region behind the triangle will drop close to zero after the diffracting of the shock, which will cause the solution blown up for most of high order schemes due to the appearance of negative density and pressure [15]. In this example, the $h$-adaptive RKDG method on Cartesian grid with positivity-preserving techniques is implemented with $\Delta x=\frac{5.6}{120}$ and $\Delta y=\frac{4.4}{60}$ combined with four levels solution refinements. The density contours and the adaptive grids at different times are illustrated in Fig. 10. These figures expose some complicated flow characteristics, such as contact discontinuities and rarefactional waves, after a right-moving shock around a finite wedge. That is justified by the line plots of Fig. 11 for the density and pressure along lines at $x=2.25$ and $y=1$, which is also demonstrates the method is capturing well with the contact discontinuities. One can observe that these results are in very good agreement with these in [15].

\section{Conclusion}

The $h$-adaptive RKDG method on Cartesian grid is developed in this paper to simulate large moving shocks. In order to suppress the failure of preserving positivity of density or pressure, a positivity-preserving limiter coupled with a modified robust HLLC flux is developed. Furthermore, in this paper, a $h$-adaptive Cartesian grid RKDG method with ghost cell immersed boundary method has been successfully developed to simulate flows through complex geometries. A reference point boundary treatment technique is presented, which can assure that the reference point locates in a fluid cell. Therefore, the cell solution polynomial of DG finite element space can be directly used as the interpolation formula to obtain the value at the reference point. Numerical examples show that the results are well matched with ones in the literature. The proposed method that has the potential to be extended to three dimensional DG discretizations, which will be our future study.

Acknowledgments The authors would like to thank the referees and editors for their helpful suggestions which improve this article. This work was partially supported by the National Science Foundation of China (Nos. 11102179, 91230110, 11328104), and PhD Studentship from De Montfort University of UK.

\section{References}

[1] G. Yang, D. M. Causon, D. M. Ingram, R. Saunders, and P. Batten, A Cartesian cut cell method for compressible flows Part A: static body problems, Aeronaut. J., vol. 101 (1997), pp. 47-56.

[2] B. SjögreEn AND N. Petersson, A Cartesian embedded boundary method for hyperbolic conservation laws, Commun. Comput. Phys., vol. 2 (2007), pp. 1199-1219. 
[3] H. ForRER AND R. JELTSCH, A higher-order boundary treatment for Cartesian-grid methods, J. Comput. Phys., vol. 140 (1998), pp. 259-277.

[4] A. DAdone A AND B. Grossman, Ghost-cell method for inviscid two-dimensional flows on Cartesian grids, AIAA J., vol. 42 (2004), pp. 2499-2507.

[5] J. M. LIU, N. ZHAO, AND O. HU, The ghost cell method and its applications for inviscid compressible flow on adaptive tree Cartesian grids, Adv. Appl. Math. Mech., vol. 1 (2009), pp. 664-82.

[6] H. Ji, F. S. LIEN, AND E. YEE, A robust and efficient hybrid cut-cell/ghost-cell method with adaptive mesh refinement for moving boundaries on irregular domains, Comput. Methods Appl. Mech. Engrg, 198 (2008), pp. 432-48.

[7] M. J. Berger, C. Helzel, AND R. J. LeVEque, H-box methods for the approximation of hyperbolic conservation laws on irregular grids, SIAM J. Numer. Anal., vol. 41 (2003), pp. 893-918.

[8] C. Helzel, M. J. Berger, And R. J. LeVeque, A high-resolution rotated grid method for conservation laws with embedded geometries, SIAM J. Sci. Comput., vol. 26 (2005), pp. 785-809.

[9] P. Colella, D. T. Graves, B. J. Keen, And D. Modian, A Cartesian grid embedded boundary method for hyperbolic conservation laws, J. Comput. Phys., vol. 211 (2006), pp. 347-366.

[10] S. K. SAMBASIVAN AND H. S. UDAYKUMAR, Ghost fluid method for strong shock interactions Part 2: Immersed solid boundaries, AIAA J. vol. 47 (2009), pp. 2923-2937.

[11] A. Chaudhuri, A. HADjADJ, AND A. Chinnayya, On the use of immersed boundary methods for shock/obstacle interactions, J. Comput. Phys., vol. 230 (2011), pp. 1731-1748.

[12] J. M. LIU, J. X. QIU, N. Zhao, O. Hu, M. Goman, And X. K. LI, Adaptive Runge-Kutta discontinuous Galerkin method for complex geometry problems on Cartesian grid, Int. J. Numer. Meth. Fluids, vol. 73 (2013), pp. 847-868.

[13] X. ZHANG AND C.-W SHU, On positivity preserving high order discontinuous Galerkin schemes for compressible Euler equations on rectangular meshes, J. Comput. Phys., vol. 229 (2010), pp. 8918-8934.

[14] X. ZHANG AND C.-W SHU, Positivity-preserving high order discontinuous Galerkin schemes for compressible Euler equations with source terms, J. Comput. Phys., vol. 230 (2011), pp. 1238-1248.

[15] X. ZHANG, Y. XIA, AND C.-W. SHU, Maximum-principle-satisfying and positivity-preserving high order discontinuous Galerkin schemes for conservation laws on triangular meshes, J. Sci. Comput., vol. 50 (2012), pp. 29-62.

[16] C. Wang, X. Zhang, C.-W. Shu, And J. G. Ning, Robust high order discontinuous Galerkin schemes for two-dimensional gaseous detonations, J. Comput. Phys., vol. 231 (2012), pp. 653-665.

[17] D. V. Kotov, H. C. Yee, And B. SJöGreen, Comparative study on high-order positivitypreserving WENO schemes, ARC-E-DAA-TN8768, SIAM Conference on Numerical Combustion, San Antonio, TX, USA, April 8th-10th, 2013.

[18] K. KONTZIALIS AND J. A. EKATERINARIS, High order discontinuous Galerkin discretizations with a new limiting approach and positivity preservation for strong moving shocks, Comput. Fluids, vol. 71 (2013), pp. 98-112.

[19] S. D. KIM, B. J. LEE, H. J. LEE, AND I. S. JEUNG, Robust HLLC Riemann solver with weighted average flux scheme for strong shock, J. Comput. Phys., vol. 228 (2009), pp. 7634-7642.

[20] B. COCKBURn AND C.-W SHU, The Runge-Kutta local projection $P^{1}$-discontinuous Galerkin 
finite element method for scalar conservation laws, Math. Model Numer. Anal., vol. 25 (1991), pp. 337-361.

[21] B. CockBuRn AND C.-W SHu, TVB Runge-Kutta local projection discontinuous Galerkin finite element method for conservation laws II: General framework, Math. Comp., vol. 52 (1989), pp. 411-435.

[22] B. CockBURN AND C.-W SHU, The Runge-Kutta discontinuous Galerkin finite element method for conservation laws V: Multidimensional systems, J. Comput. Phys., vol. 141 (1998), pp. 199-224.

[23] B. CockBuRn, S. Y. Lin, AND C.-W SHU, TVB Runge-Kutta local projection discontinuous Galerkin finite element method for conservation laws III: One dimensional systems, J. Comput. Phys., vol. 84 (1989), pp. 90-113.

[24] B. Cockburn, S. Hou, AND C.-W Shu, The Runge-Kutta local projection discontinuous Galerkin finite element method for conservation laws IV: The multidimensional case, Math. Comp., vol. 54 (1990), pp. 545-581.

[25] C.-W SHU AND S. OsHER, Efficient implementation of essentially non-oscillatory shockcapturing schemes, J. Comput. Phys., vol. 77 (1988), pp. 439-471.

[26] M. Berg, O. Cheong, M. Kreveld, and M. Overmars, Computational Geometry: Algorithms and Applications, Springer-Verlag, Berlin Heidelberg, 2008.

[27] T. CECIL, S. OsHeR, AND J. QIAN, Essentially non-oscillatory adaptive tree methods, J. Sci. Comput., vol. 35 (2008), pp. 25-41.

[28] H. Q. ZHU AND J. X. QIU, An h-adaptive RKDG method with troubled-cell indicator for two-dimensional hyperbolic conservation laws, Adv. Comput. Math., vol. 39 (2013), pp. 445-463.

[29] E. F. Toro, Riemann Solvers and Numerical Methods for Fluid Dynamics, Berlin Heidelberg, Springer, 1999.

[30] E. F. TORo, M. Spruce, AND W. SPEAREs, Restoration of the contact surface in the HLL Riemann solver, Shock Waves, vol. 4 (1994), pp. 25-34.

[31] A. HARTEN, P. D. LAX, AND B. VAN LeER, On upstream differencing and Godunov-type schemes for hyperbolic conservation laws, SIAM Rev., vol. 25 (1983), pp. 35-61.

[32] P. Batten, N. Clarke, C. LAMbert, AND D. M. CAuson, On the choice of wavespeeds for the HLLC Riemann solver, SIAM J. Sci. Comput., vol. 18 (1997), pp. 1553-1570.

[33] D. H. WANG, N. ZHAO, O. HU, AND J. M. LiU, A ghost fluid based front tracking method for multimedium compressible flows, Acta. Math. Sci., vol. 29 (2009), pp. 1629-1646.

[34] L. Krivodonova, J. Xin, J. F. Remacle, N. Chevaugeon, AND J. E. Flaherty, Shock detection and limiting with discontinuous Galerkin methods for hyperbolic conservation laws, Appl. Numer. Math., vol. 48 (2004), pp. 323-338.

[35] D. De ZeEuw, A Quadtree-Based Adaptively-Refined Cartesian-Grid Algorithm for Solution of the Euler Equations, PhD Thesis, University of Michigan, 1993.

[36] X. Y. HU, N. A. AdAMS, AND C.-W SHU, Positivity-preserving method for high-order conservative schemes solving compressible Euler equations, J. Comput. Phys., vol. 242 (2013), pp. 169-180.

[37] J. Lee and S. M. Ruffin, Development of a Turbulent Wall-Function Based Viscous CartesianGrid Methodology, AIAA Paper 07-1326. 45th AIAA Aerospace Sciences Meeting and Exhibit, Reno, Nevada, 2007. 